\title{
Quelques exemples d'interaction fluide-structure dans les réacteurs nucléaires
}

\author{
Some examples of fluid-structure interactions in nuclear reactors
}

\author{
par Laurent Borsoi \\ Commissariat à l'Energie Atomique (CEA), Direction des Réacteurs Nucléaires \\ Laboratoire d'Etudes de Dynamique
}

Having gone through the specificity of nuclear energy beforehand, this article deals with the issue of FluidStructure Interaction (FSI) in nuclear reactors. In this respect, it makes use of some cases taken out of the French electro-nuclear project. Bearing these examples in mind, it ends with a prospective elaboration of the needs of FSI in 10 years time.

\section{I — SPÉCIFICITÉ DU NUCLÉAIRE}

Les problèmes d'Interaction Fluide-Structure (IFS) rencontrés dans les réacteurs nucléaires sont-ils spécifiques? Et si oui, en quoi ? La réponse à ces questions est évidemment nuancée.

Comme pour beaucoup d'équipements industriels, I'IFS (sous-entendu au sens mécanique) ne se situe pas dans les préoccupations premières des concepteurs de réacteurs. Cela ne signifie pas qu'ils ignorent ce type de phénomènes, et leurs conséquences, mais ils ont tout simplement d'autres priorités dans l'élaboration de machines aussi complexes. Un réacteur nucléaire civil est avant tout une machine thermique, d'où la primauté portée sur les transferts thermiques qu'il convient d'optimiser : source chaude (nature du cœur et de son combustible, température,...), fluides caloporteurs (pour extraire la puissance thermique dégagée par le cœur, la transporter, l'échanger), structures (pour contenir le cœur, faire circuler et canaliser les fluides, favoriser les échanges thermiques). Ce processus d'optimisation se trouve évidemment grandement compliqué par la spécificité nucléaire qui ajoute de très fortes contraintes, notamment par la Neutronique (présence d'un modérateur par exemple) et la Sûreté d'une manière générale.

La Sûreté nucléaire a trois objectifs :

1. assurer le fonctionnement normal de l'installation en limitant les rejets d'effluents radioactifs dans l'environnement,

2. prévenir des incidents ou accidents,

3 . limiter les conséquences d'éventuels incidents ou accidents.

La philosophie de la défense en profondeur consiste grossièrement à faire tout ce qu'il faut pour que "cela " (un incident ou accident) n'arrive pas, mais que si « cela " arri- vait quand même, il existe un moyen de maîtriser la situation (i.e. contrôler la réactivité, évacuer l'énergie dégagée par le combustible, confiner les produits radioactifs), et de ne pas passer à une situation plus dégradée. La Sûreté nucléaire impacte ainsi I'IFS à plusieurs niveaux, notamment par :

- la justification de la tenue mécanique du matériel en fonctionnement normal (prévention),

- la prise en compte dans le dimensionnement d'accidents hypothétiques tels des séismes de fortes intensités, des ruptures d'enceinte sous pression, des explosions, etc., (limitation des conséquences).

Il n'est besoin d'insister sur la complexité des réacteurs nucléaires composés des circuits fluides principaux, assurant les transferts thermiques et focalisant généralement l'attention, mais également d'une myriade de circuits et composants auxiliaires nécessaires aux fonctionnements normal, incidentel, voire accidentel du réacteur : systèmes de régulation, de protection, de sauvegarde, parades, etc. Cette complexité fait que même les réacteurs les plus standardisés peuvent comporter, lors de leurs évolutions (nouveau mode de fonctionnement, nouvelle série, changement apparemment mineur,...), de nouvelles configurations d'IFS pour lesquelles il n'existe aucun retour d'expérience. Inutile de préciser que ce manque de retour d'expérience se trouve encore plus accru pour un réacteur prototype au dessin complètement nouveau.

En résumé, un réacteur nucléaire est une machine thermique formidablement complexe, présentant une multitude de sous-ensembles aux dessins très variés et devant satisfaire un niveau d'exigence très élevé de Sûreté. Schématiquement I'IFS y est plutôt " subie " - les ingénieurs s'en passeraient bien ! - et est étudiée à différents stades de la vie de l'installation : 
- dans la phase initiale de conception, plus au stade de la vérification du dimensionnement qu'au stade du pré-dimensionnement, sauf pour certains cas simples à traiter et que l'on sait critiques,

- durant la vie de l'installation, de manière prédictive, suite à des modifications de matériels ou de conditions de fonctionnement, voulues (amélioration des rendements, extension de la durée de vie,...), ou non (fonctionnement en situation dégradée),

- durant la vie de l'installation, suite à des incidents non prévus pour tenter de les expliquer: c'est le fameux retour d'expérience, seul « juge de paix » dans l'industrie, et qui met parfois en évidence des phénomènes extrêmement subtils.

\section{II 口 OBJET DE L'ARTICLE}

Ce colloque sur l'IFS ayant pour but de stimuler les échanges entre spécialistes de différents domaines (Mécanique, Génie Civil, Aéronautique,...), cet article essaye d'illustrer la problématique propre au Nucléaire via quelques exemples tirés du programme électronucléaire français. Ces exemples ne sont pas traités en profondeur - ce n'est pas l'objet de cet article - mais toujours placés en perspective. On s'en inspire pour finalement tenter une ébauche de prospective à 10 ans des besoins en IFS (2000-2010).

Le choix du programme électronucléaire français réduit les réacteurs étudiés aux seuls RNR (Réacteur à Neutrons Rapides: Phénix et Superphénix) et REP (Réacteur à Eau sous Pression : 58 unités en France en 99). Il n'est donc pas question de prétendre à l'exhaustivité, mais à la seule illustration. (NB : sur environ 450 réacteurs électronucléaires dans le monde, presque la moitié sont des REP). Bien qu'elles soient sémantiquement discutables, on adopte dans cet article les définitions suivantes [1] :

Couplage fluide-structure. Il s'agit là de traiter l'interaction dynamique entre une structure vibrante et un fluide au repos (i.e. le fluide est immobile si l'on excepte le flot oscillant créé par la structure vibrante). Les phénomènes physiques impliqués dans un tel couplage concernent principalement la masse ajoutée due à l'inertie du fluide, et, à un degré moindre, l'amortissement supplémentaire créé par la viscosité du fluide (phénomène parfois prépondérant en cas de lames fluide minces).

Excitation turbulente. Elle résulte des fluctuations aléatoires inévitablement présentes dans tout fluide industriel. En monophasique, ces fluctuations sont une conséquence directe de la turbulence développée dans le fluide. En diphasique, la situation est moins claire puisqu'à la turbulence de chaque phase s'ajoutent les fluctuations du mélange lui-même. L'excitation appliquée à la structure, et générée par le fluide. est généralement caractérisée par un spectre large bande, relativement plat, dont l'énergie est confinée en dessous d'une certaine fréquence de coupure. Par ailleurs des pics, dus à des phénomènes de détachements tourbillonnaires, peuvent également se superposer à ce spectre. Dans ce cas l'expression « excitation turbulente "n'est plus réellement appropriée, si ce n'est par souci de rassembler dans cette classe toute excitation forcée produite par le fluide et totalement indépendante du mouvement de la structure.

Interaction Fluide-Structure (IFS). Il s'agit là d'analyser toutes les interactions entre un fluide sous écoulement et une structure vibrante. Quand la structure est élastique, ces interactions se traduisent par des forces dites « fluidélastiques». L'IFS conduit à un échange d'énergie entre l'écoulement et la structure. Dans certains cas, ce processus non-conservatif a un effet stabilisant favorable en engendrant, par exemple, de l'amortissement additionnel au système couplé vibrant. Mais souvent il a un effet contraire en induisant des instabilités fluidélastiques qui résultent en des vibrations excessives. Dans l'hypothèse linéaire fluidélastique, ces vibrations croissent sans cesse (explosion exponentielle). Dans la réalité, elles peuvent parfois s'auto-limiter par les non-linéarités, structurelles ou fluides, induites par les fortes amplitudes (impacts, plasticité, recirculation favorable, etc.). Les instabilités fluidélastiques sont toujours liées aux variations des caractéristiques dynamiques (fréquence, amortissement) du système couplé fluide-structure avec la vitesse de l'écoulement. Quand cette vitesse atteint sa valeur critique, il y a instabilité par "divergence" (fréquence propre nulle suite à une perte totale de raideur) ou par «flottement » (amortissement devenant négatif, confusion de fréquences). Les phénomènes de détachements tourbillonnaires avec accrochage pourraient également se ranger dans cette classe IFS, puisqu'elle regroupe - par opposition avec la « turbulence » - toute excitation produite par le fluide et dépendante du mouvement de la structure.

\section{III a INSTABILITÉ DU DÉVERSOIR DE SUPERPHÉNIX}

Superphénix, situé à Creys-Malville, est un Réacteur à Neutrons Rapides (RNR), de type piscine, de $1300 \mathrm{MWe}$ refroidi avec du sodium liquide. Des viroles minces disposées à l'intérieur du bloc réacteur séparent le sodium en différents volumes nommés « collecteurs ». Les principaux collecteurs sont de grande capacité comme le collecteur chaud en sortie du cœur ou le collecteur froid en aspiration des pompes primaires. Mais d'autres, comme les collecteurs d'alimentation et de restitution du circuit de refroidissement de la cuve principale, constituent en fait des lames fluide minces vu leurs dimensions (voir fig. la).

Durant les premiers tests hydrauliques du réacteur, en octobre 1984, des vibrations inattendues de grandes amplitudes furent détectées grâce au système de surveillance vibratoire mis en place. Leur signature changeait selon le débit primaire, mais montrait une nette composante basse fréquence à 0,3-0,4 Hertz. La virole-déversoir séparant les collecteurs d'alimentation et de restitution du circuit de refroidissement de la cuve principale, était particulièrement affectée.

Chargé de l'investigation, le CEA/Saclay mit aussitôt en évidence un subtil phénomène d'instabilité fluidélastique. dont il donna en quelques semaines, via une maquette analytique très simplifiée (plane) et de premières simulations numériques, un modèle satisfaisant capturant l'essentiel de la physique. Ce phénomène est grossièrement schématisé figure $1 b$. Au lieu d'être régulier, l'écoulement du sodium au dessus du déversoir se produit de manière discontinue et pulsée suite au mouvement latéral de la virole, lui-même entretenu par ce flot pulsé, notamment par l'impact des "bouffées » de sodium tombant du déversoir. La gravité, ainsi que la souplesse de la virole-déversoir permettant une rétroaction du collecteur de restitution vers le collecteur d'alimentation, produisent donc ce phénomène d'auto-excitation, nettement plus complexe dans la réalité car impliquant toutes sortes de modes couplés fluide-structure, notamment de ballottement.

Le modèle considère, i) au niveau du déversoir, une source de débit négative : $\pm q_{d}(t, \theta)= \pm k \sqrt{g} h(t, \theta)^{3 / 2}$, $h$ étant la hauteur de la lame fluide $(\geq 0)$ se déversant à l'azimut $\theta$, et $k$ une constante propre au déversoir; ii) au niveau du collecteur de restitution, une source de débit positive : $q_{\mathrm{r}}(\mathrm{t}, \theta)=+\mathrm{q}_{\mathrm{d}}(\mathrm{t}-\tau, \theta), \tau$ étant un temps de retard dû à la chute du sodium, ainsi qu'une source de pression : $p(\Delta \mathrm{z})$ $=\rho g \Delta z+q_{r} V_{p} / e_{r}$, localisée à la côte $\Delta z$ sous la surface libre, $V$ étant la vitesse du sodium «impactant » et $\mathrm{e}_{\mathrm{r}}$ la largeur du collecteur en ce point. Ces sources sont introduites comme excitation du système couplé fluide-structure de l'ensemble des deux collecteurs et de la virole-déversoir. La résolution s'effectue par synthèse modale. 


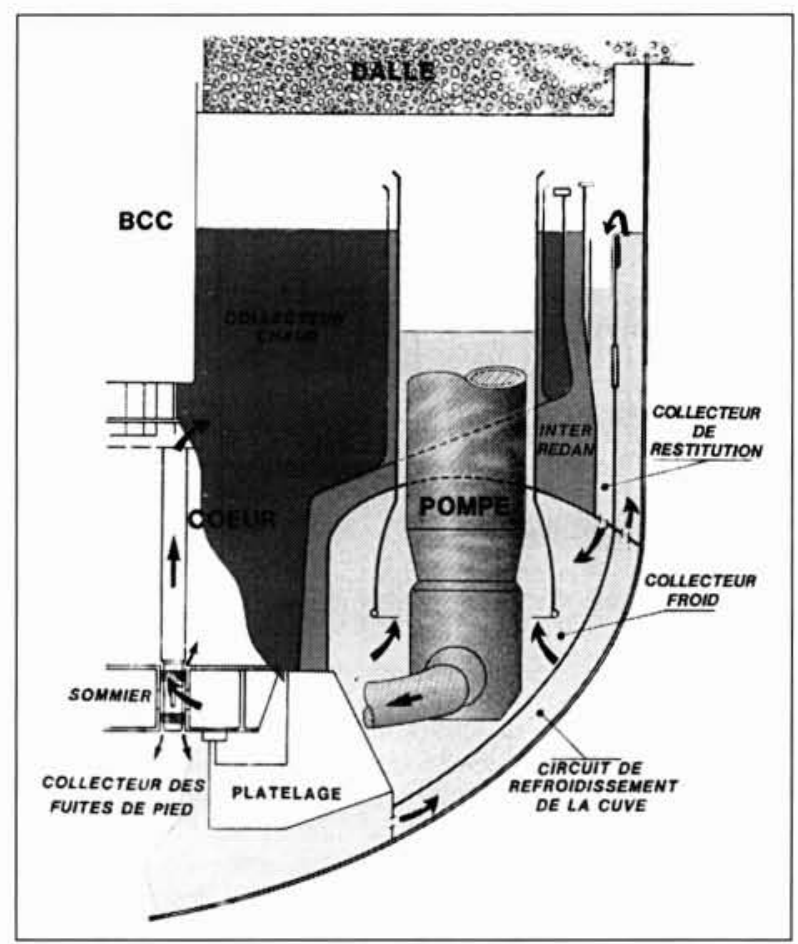

1a. Circuit primaire (vue partielle) de Superphénix.

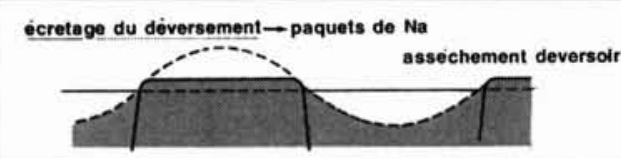

1c. Ecoulement pulsé.

Dans un $1^{\mathrm{er}}$ temps, les équations linéarisées permirent d'établir des domaines de stabilité sur un diagramme hauteur de chute - débit total déversant. Il apparut ainsi que deux types d'instabilité pouvaient se développer :

1. des instabilités de flottement par confusion de fréquences de deux modes proches de ballottement des collecteurs couplés par la virole (modes à basse fréquence d'indice azimutal $n$ ),

2. des instabilités de flottement par amortissement négatif de modes de virole (à plus haute fréquence).

Dans un $2^{\mathrm{e}}$ temps, des synthèses modales non-linéaires furent réalisées afin d'analyser les cycles limites susceptibles d'être atteints en régime instable [2].

D'une manière générale, le modèle et les cartes de stabilité qui en découlaient, furent recoupés par les expériences menées sur les trois maquettes analytiques construites pour la circonstance (la plane évoquée ci-dessus, deux cylindriques, l'une au 1/12 au CEA puis une autre au 1/4 à EDF) et les campagnes d'essais menées in-situ sur Superphénix. L'instabilité observée lors des essais hydrauliques était du $1^{\text {er }}$ type. Cette compréhension des phénomènes physiques permit de prédire «à coup sûr " les régimes de fonctionnement du circuit de refroidissement de la cuve de Superphénix pour lesquels il n'y aurait aucun risque d'instabilité. Il fut alors possible d'atteindre ces régimes en ajustant le débit de refroidissement par simple modification de pertes de charge en pied d'assemblages. Cette modification, d'un coût mineur, n'eut aucune incidence sur les performances du réacteur ni sur le déroulement de sa mise en service.

Les enseignements de cet incident sont de trois ordres. I) Faute de retour d'expérience, et «obnubilés " par les problèmes vibratoires potentiels des circuits principaux (qui
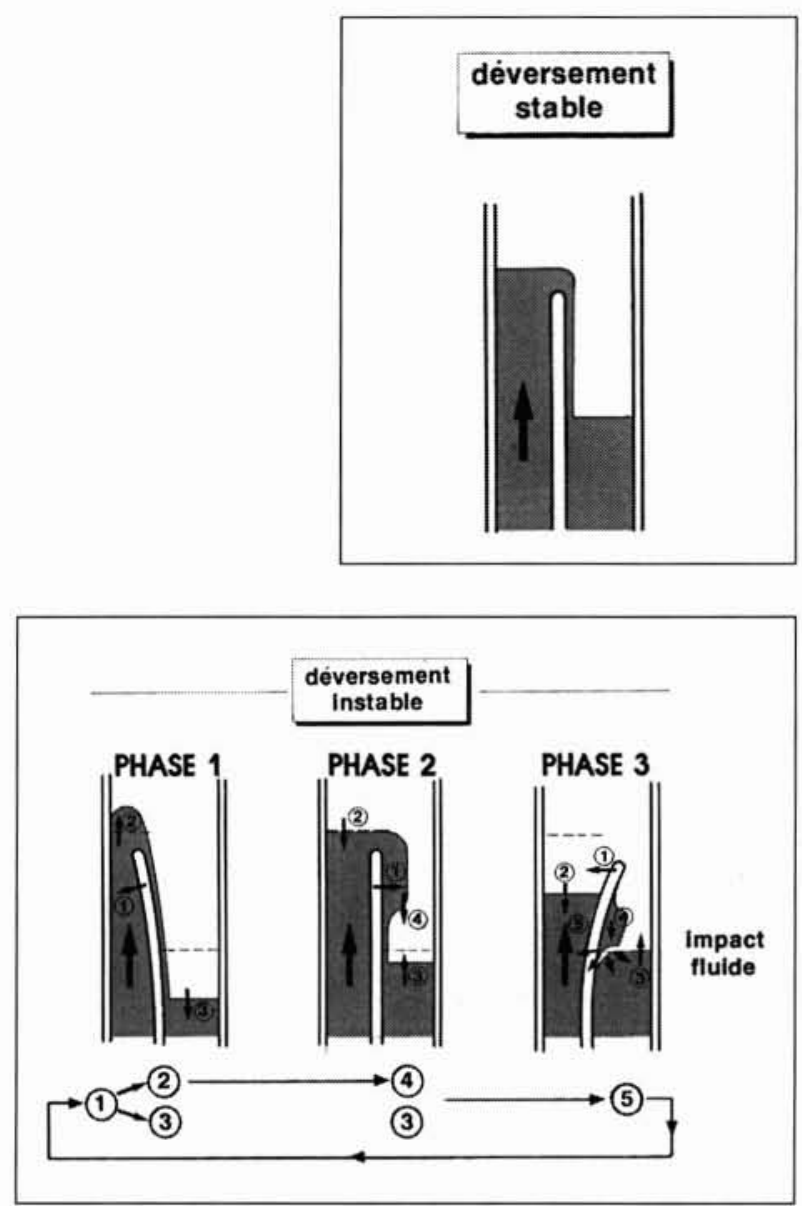

1b. Mécanisme d'instabilité (schématisé) du déversoir.

donnèrent lieu à de nombreuses études), les ingénieurs-dynamiciens "oublièrent " d'analyser ce système annexe de déversoir. Par ailleurs l'auraient-ils fait, que peut-être seraient-ils passés à côté, vu la subtilité du phénomène. II) Une fois le problème découvert, les équipes ont fait preuve d'une étonnante réactivité, due en partie à leur expérience en IFS et la disponibilité d'outils numériques traitant du fluide-structure. III) Le remède industriel fut étonnamment simple et peu coûteux. Cela n'est pas toujours le cas, mais pourrait être considéré comme la contrepartie favorable de la subtilité ci-dessus évoquée : grande sensibilité des paramètres $\Rightarrow$ " subtilité » du phénomène $\Rightarrow$ anticipation difficile $\Rightarrow$ occurrence $\Rightarrow$ compréhension (souvent difficile) $\Rightarrow$ remède "facile " par légère modification de paramètre(s) (avec notamment les phases d'assèchement - voir fig. Ic).

\section{GIBRATIONS DES FAISCEAUX DE TUBES DES GÉNÉRATEURS DE VAPEUR}

Les Générateurs de Vapeur (GV) REP assurent le transfert thermique du circuit primaire au circuit secondaire (environ $1 \mathrm{GW}$ par GV). L'eau primaire, sous pression et réchauffée par les réactions nucléaires du cœur $\left(155 \mathrm{bar}, 320^{\circ} \mathrm{C}\right)$ circule à l'intérieur de milliers de tubes en $U$ (inversé) d'environ $20 \mathrm{~mm}$ de diamètre, $1 \mathrm{~mm}$ d'épaisseur et $20 \mathrm{~m}$ de long. Au contact de ces tubes, et remontant le long de ceux-ci, l'eau secondaire se réchauffe et se vaporise $\left(60 \mathrm{bar}, 280^{\circ} \mathrm{C}\right)$. La vapeur ainsi produite est alors séchée, puis envoyée vers les 
turbo-alternateurs. L'enveloppe du circuit primaire constitue la $2^{\mathrm{e}}$ barrière de confinement (la $1^{\mathrm{re}}$ étant la gaine des crayons combustible) et doit ainsi être parfaitement étanche pour des raisons de Sûreté. Or elle est constituée à $80 \%$ de la surface d'échange des tubes GV. Ceux-ci sont donc particulièrement surveillés. Ils sont potentiellement sujets à des vibrations sous écoulement vu leur exposition au flot externe secondaire, transverse et monophasique (eau) en bas du faisceau, axial et diphasique (eau-vapeur) le long, transverse et diphasique (eau-vapeur à $85 \%$ vol.) au sommet dans la région des cintres («chignon»). Des plaques entretoises régulièrement espacées maintiennent fermement les tubes dans leur partie droite. Dans la zone du chignon, la plus critique, des barres anti-vibratoires sont insérées entre eux pour assurer un certain maintien (Voir fig. 2).

Contrairement au déversoir de Superphénix au dessin unique, les échangeurs de chaleur à faisceaux de tubes sont des équipements courants dans l'industrie. Dès la fin des années 50 , on savait que ces faisceaux soumis à un fort écoulement externe transverse pouvaient devenir instables. Sous la poussée, en particulier, du Nucléaire avec son exigence de Sûreté, de nombreuses études expérimentales et théoriques commencèrent à être menées dans les années 70 dans le but de comprendre, modéliser, prédire, éviter... La complexité des phénomènes avait alors été sous-estimée, et bien que d'incontestables progrès aient été accomplis, il faut bien admettre que, 30 ans après, le problème reste largement ouvert [3].

Connors, de Westhinghouse, proposa dès 1970 un modèle de l'instabilité fluidélastique des faisceaux de tubes sous

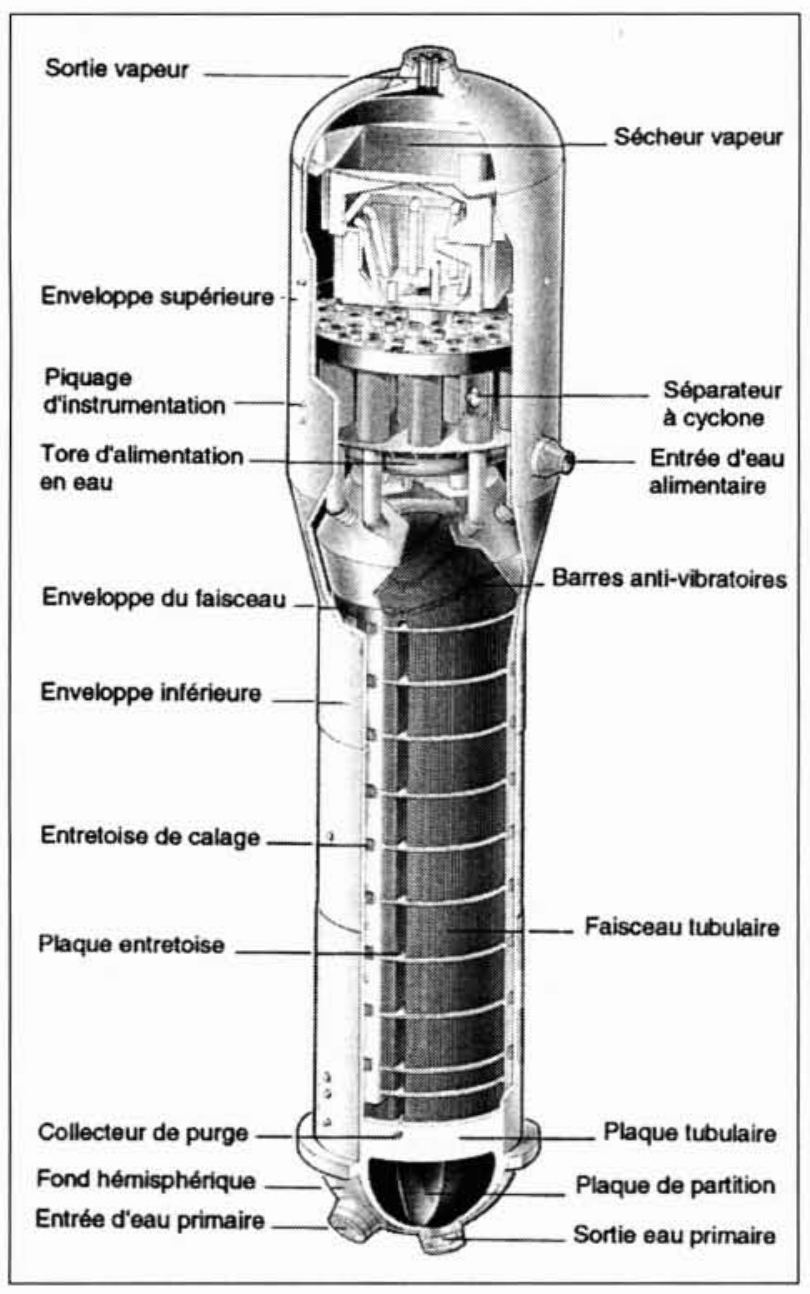

2. Générateur de Vapeur REP. écoulement transverse, à partir d'expériences conduites sur une rangée de tubes rigides sous écoulement d'air. En déplaçant statiquement un tube dans la direction de l'écoulement $(x)$, il détecta et mesura sur le tube voisin une force induite perpendiculairement $(y)$; et réciproquement en déplaçant un tube suivant $y$, il mesura une force induite sur le tube voisin suivant $x$. Introduites dans le système dynamique des deux tubes, dont les caractéristiques sont très proches par construction (densité linéique $\mathrm{m}$, diamètre $D$, pulsation propre $\omega_{0}$ ), ces forces de couplage statique produisent un amortissement modal diminuant avec la vitesse $V$ de l'écoulement : $\xi(V)=\xi_{(V=0)} \pm k \rho V^{2} / m \omega_{0}^{2}, k$ étant une constante liée au couplage, $\rho$ la masse volumique du fluide. A la vitesse critique $V_{c}$, le système devient instable par perte d'amortissement; sous forme adimensionnel : $\frac{V_{c}}{f_{0} D}=K \sqrt{\frac{2 \pi \xi_{0} m}{\rho D^{2}}}$, soit $V_{r c}=K \cdot \sqrt{A_{r}}$ avec $K$ la constante de Connors (différente du $\mathrm{k}$ précédent) et $A_{r}$ le paramètre réduit masse-amortissement (nombre de Scruton).

Il s'en suivit durant les années 70-80 une frénésie d'expériences dans le monde entier, chaque laboratoire mesurant «sa " constante de Connors pour telle ou telle configuration d'essais (géométrie du faisceau et nature de l'écoulement). La compilation de toutes ces données sur des « cartes de Connors $"\left(\mathrm{~A}_{\mathrm{r}} ; \mathrm{V}_{\mathrm{rc}}\right)$ permit d'établir des domaines de stabilité « garantie " pour les faisceaux, données cruciales pour la conception.

Le succès, toujours actuel, du modèle de Connors, ne doit pas masquer ses limites. A la fin des années 70 , on mit en évidence, notamment au CEA/Saclay, la possibilité de départ en instabilité d'un seul tube flexible placé dans un faisceau de tubes rigides. La physique du modèle de Connors, qui s'appuyait sur un couplage quasi-statique d'au moins deux tubes, tombait donc, ce qui relança l'effort de recherche. On se focalisa alors sur ce cas du tube flexible unique noyé dans une «forêt rigide". On exprima la force fluidélastique qui s'exerce sur ce tube en fonction de son déplacement, mais surtout de sa vitesse, cette contribution étant prépondérante. En considérant un certain retard $\tau$ dans l'établissement de cette force - ce qui paraît intuitif : l'écoulement perturbé mettant un «certain temps " à se réorganiser - on retrouvait, par le déphasage ainsi introduit dans la force d'amortissement, une possibilité d'instabilité. Les modèles proposés dans cette voie (Price-Paidoussis, Lever-Weaver, GrangerPaidoussis,...) marquent certes un progrès, mais sont toujours loin d'être prédictifs vu les constantes à ajuster au cas par cas. Par ailleurs on continue aujourd'hui à compiler les résultats expérimentaux sur des cartes de Connors même si on sait ce formalisme adimensionnel inadéquat, ou tout du moins trop simpliste et pas assez discriminant. (NB. La racine y est parfois remplacée par un exposant $n \neq 0,5$ ).

Les travaux expérimentaux des années 70 et 80 portaient principalement sur la détermination de la vitesse critique d'instabilité $V_{c}$ des faisceaux. Une tendance est apparue dans les années 90 , consistant à mesurer les forces fluidélastiques sur toute la plage des vitesses d'écoulement $V<V_{c}$, et même maintenant $\mathrm{V} \geq \mathrm{V}_{\mathrm{c}}$. En écrivant le système dynamique d'IFS : $m_{s} \cdot \ddot{x}+c_{s} \cdot \dot{x}+k_{s} \cdot x=f_{t} \pm\left(m_{f} \cdot \ddot{x}+c_{f} \cdot \dot{x}+k_{f} \cdot x\right)$, avec $\mathrm{x}$ le déplacement de la structure, l'indice $s$ pour structure, $f$ pour fluide, $\mathrm{m}$ la masse, $\mathrm{c}$ l'amortissement, $\mathrm{k}$ la raideur, et $\mathrm{f}_{t}$ les forces fluide turbulentes, les coefficients fluidélastiques à identifier sont $m_{f}, c_{f}, k_{f}$. En général, la masse ajoutée $m_{f}$ ne dépend pas de la vitesse d'écoulement et est ainsi facile à déterminer. A l'opposé, les termes d'amortissement et de raideur dépendent de cette vitesse (c'est la raison de l'instabilité) et requièrent des méthodes d'identification, d'autant plus sophistiquées que le système d'IFS présente des modes proches couplés (cas des faisceaux souples) et que l'excita- 
tion turbulente est inconnue. La stratégie globale des coefficients fluidélastiques pourrait se résumer comme suit : i) identification expérimentale à l'aide de maquette analytique, ii) réduction adimensionnelle, iii) intégration des coefficients réduits dans des codes afin de calculer des situations réelles. Avec cette approche les vibrations sous écoulement peuvent être estimées sur toute la gamme des vitesses: $\mathrm{V}<<\mathrm{V}_{\mathrm{c}}$ (peu d'IFS), $V<V_{c}$ (IFS notable) et même $V \geq V_{c}$ (instabilité due à l'IFS mais contrôlée par des non-linéarités comme des impacts). L'estimation des dommages, comme la fatigue élastique ou l'usure vibratoire, s'en suit.

\section{V —MODÉLISATION SOUS SÉISME DES CCEURS DE RÉACTEURS}

Pour des raisons évidentes de Sûreté, le séisme fait partie des agressions externes prises en compte dans le dimensionnement des installations nucléaires. La présence de fluides denses dans les réacteurs doit ainsi être modélisée. D'après les définitions du chapitre II, cela se réduit à un "simple " problème de couplage fluide-structure quand les vitesses d'écoulement peuvent être négligées. L'effet prépondérant est alors inertiel : le séisme secoue la structure, qui ellemême "pousse " le fluide contenu à l'intérieur. Beaucoup de configurations, comme par exemple une tuyauterie remplie d'eau, ne soulèvent aucune difficulté de modélisation, la masse ajoutée par le fluide se ramenant simplement à la masse embarquée. Cela n'est plus le cas pour des structures plus complexes, comme les cours des réacteurs, comprenant de nombreux composants internes immergés. Chaque élément de paroi vibrante, en «poussant " le fluide non-visqueux devant lui, impose une condition aux limites au fluide : $\partial p / \partial n=-\rho \ddot{u}_{n} ;$ par ailleurs celui-ci, considéré comme incompressible, satisfait à l'équation : $\Delta p=0$ (avec $u_{n}$ le déplacement normal de la paroi, $\mathrm{p}$ la pression induite). Après résolution, les pressions en paroi donnent les forces de couplage fluide-structure, celles-ci se traduisant dans la modélisation par une matrice masse ajoutée aux termes extra-diagonaux non nuls. La masse du fluide embarqué est bien évidemment retrouvée via cette matrice dont chaque terme $m_{i j}$ permet d'exprimer la force exercée par le fluide sur le degré de liberté i de la structure sous l'effet d'une accélération du ddl $\mathrm{j}: \mathrm{f}_{\mathrm{i}}=\mathrm{m}_{\mathrm{ij}} \ddot{\mathrm{x}}_{\mathrm{j}}$, mais ne représente pas une masse physique. Les termes $\mathrm{m}_{\mathrm{ij}}$ peuvent ainsi être négatifs et très grands, en particulier en cas de confinement. Il convient donc de traiter tous les termes de la matrice avec soin, notamment en cas de séisme où une partie de l'excitation peut être transmise aux structures internes directement par le fluide. Le fluide étant incompressible, l'effet des masses ajoutées est instantané. La compressibilité du fluide doit, et peut, être prise en compte dans certaines applications ; les masses ajoutées deviennent alors fréquentielles [4].

La modélisation sous séisme des REP et RNR a fait l'objet de nombreux développements. Traiter le couplage fluidestructure était notamment crucial pour Superphénix vu les grandes viroles souples et la présence du sodium. La modélisation des cœurs REP et RNR pose en fait autant de problèmes pratiques que théoriques: i) l'excitation sismique transmise au cœur par les structures le soutenant doit être correctement évaluée, ii) le cœur lui-même est constitué d'un ensemble d'assemblages généralement non-linéaires et pouvant choquer entre eux, iii) la prise en compte du fluide doit être compatible avec la modélisation forcément simplifiée du cœur. De nombreux essais ont été conduits sur table vibrante (maquette à échelle réduite ou même spécimen à échelle 1) afin de conforter la modélisation des cœurs sous séisme. Des méthodes d'homogénéisation ont notamment été développées et testées. Les glissements de fréquences dus à la présence du fluide peuvent atteindre $-30 \%$, ce qui illustre l'importance des masses ajoutées.
Le second effet du couplage fluide-structure, moins important que l'effet inertiel, est l'amortissement dû à la viscosité du fluide. Cet amortissement, assez faible, est souvent pris de manière forfaitaire, recalé d'après des expériences. L'écoulement peut aussi générer de l'amortissement, positif ou négatif dans le cas général de l'IFS. Les essais montrent par exemple que les assemblages combustible REP sont fortement amortis sous l'effet de l'écoulement primaire axial, à des taux pouvant dépasser $20 \%$.

L'IFS affecte aussi le pincement de lames fluide suite à des impacts [4]. Sous l'action du séisme, tous les assemblages combustible d'une rangée REP s'empilent les uns contre les autres et chargent fortement la grille médiane du $1^{\text {er }}$ assemblage contre le cloisonnement. L'évacuation du fluide "écrasé " tend à diminuer l'effort subi par la grille :

$$
F_{f}=\lambda\left[ \pm \alpha(\ddot{x} / x)+\beta\left(\dot{x}^{2} / x^{2}\right) \pm \gamma \nu\left(\dot{x} / x^{3}\right) \pm 1,5 \zeta\left(\dot{x}|\dot{x}| / x^{2}\right)\right] \text {. }
$$

avec $x$ la largeur de la lame fluide, $\lambda$ une constante liée à la géométrie de la grille et à la masse volumique du fluide, $v$ sa viscosité, $\alpha, \beta, \gamma, \zeta$ des paramètres à identifier d'après essais. Cette force est sensible aux circulations réelles sous la grille (planéité des surfaces, présence d'orifices,...), ainsi qu'à l'écoulement axial primaire.

\section{VI —USURE DES GRAPPES ET DE L'INS- TRUMENTATION-CEUR DANS LES CUVES REP}

Comme déjà signalé, les réacteurs nucléaires comportent une multitude de composants connexes pour assurer leur bon fonctionnement. Beaucoup de ces composants internes sont constitués de structures tubulaires élancées, généralement flexibles vu leurs géométrie et système de supports qui comporte des jeux pour des raisons d'assemblage, de guidage et d'expansion thermique. De telles structures filaires, isolées ou arrangées en faisceau, peuvent être soumises à des écoulements externes, axiaux ou transverses, de grandes vitesses vu les puissances thermiques à transférer, et ainsi sujettes à des phénomènes d'IFS au sens large. Certaines configurations, que l'on sait potentiellement critiques, sont maintenant l'objet d'attention dès la conception, ce qui n'a pas toujours été le cas pour les $1^{\text {er }}$ réacteurs. On veille par exemple à la non-coïncidence des fréquences de détachement tourbillonnaire avec les fréquences propres des structures en raidissant généralement ces dernières $\left(S_{r}=f . D / V>>0,2\right.$ pour un cylindre en régime sous-critique par ex.). Mais la plupart des configurations ne se prêtent pas à des analyses aussi simples. Et bien des phénomènes liés à l'IFS ne sont en fait révélés que par le retour d'expérience suite à une surveillance vibratoire ou au travers de dommages mis en évidence. Ces dommages peuvent être "foudroyants" (ruine par fatigue oligocyclique par ex.) et détectés dès les premiers tests de fonctionnement. Mais d'autres peuvent demander une période d'incubation plus ou moins longue comme la fatigue élastique ou l'usure-vibratoire, et n'être détectés qu'après des mois, voire des années de fonctionnement.

\subsection{Les grappes de commande}

Les réactions nucléaires dans un REP sont contrôlées (entre autres) par les grappes de commande, constituées chacune de 24 crayons absorbants, " neutrophages ", suspendus à une « araignée ", et s'insérant dans les tubes-guides des assemblages combustible. L'araignée est connectée à une barre de commande, que l'on déplace pas à pas pour des contrôles fins de réactivité, ou que l'on libère en cas d'arrêt d'urgence. Dans ce cas l'ensemble (grappe + barre) chute par gravité en $1,5 \mathrm{~s}$ environ. Les crayons absorbants sont longs d'environ 
$4 \mathrm{~m}$ (la hauteur du combustible) pour un diamètre de $10 \mathrm{~mm}$, et sont donc très flexibles. En fonctionnement normal à pleine puissance, les grappes sont en position haute. Les crayons sont alors maintenus latéralement par le haut de l'assemblage combustible, un guidage continu constitué de fourreaux et tubes fendus sur environ $1 \mathrm{~m}$, un guidage discontinu constitué de "cartes » espacées de $300 \mathrm{~mm}$, tous ces supports comprenant des jeux (de l'ordre de $0.5 \mathrm{~mm}$ ). Les guidages continu et discontinu sont intégrés dans des carters qui, outre leur fonction mécanique, canalisent les différents écoulements. En particulier l'eau primaire venant de l'assemblage, rentre directement dans le guidage continu pour en sortir via des ouïes latérales et rejoindre, au travers le plenum supérieur, les tuyauteries primaires chaudes. Par ailleurs, le guidage discontinu est le siège d'écoulements de recirculation du primaire ou créés par différents by-pass. Les crayons des grappes peuvent ainsi être sujets à des vibrations sous écoulement. (Voir fig. 3).

En 1986, une concentration anormale d'argent fut détectée dans le circuit primaire d'un $900 \mathrm{MWe}$, dont l'origine était le percement d'un crayon absorbant après quatre années de fonctionnement. Les investigations menées mirent rapidement en évidence un phénomène généralisé "d'usure-souscarte ", non suspecté jusqu'alors - on craignait principalement les usures longitudinales dues au mouvement pas à pas des grappes. Cette usure localisée est inacceptable dans le sens où elle fragilise structurellement le crayon qui risque de casser, et ainsi d'empêcher la grappe de chuter (ce qui effectivement se produisit quelques fois). Face à ce problème de sûreté, il s'en suivit une multitude d'actions de la part d'EDF, de Framatome et du CEA qu'il n'est pas possible de relater ici. On notera seulement : i) l'adoption immédiate de dispositions opérationnelles pour pouvoir fonctionner avec ce problème : étalement de l'usure (en déplaçant les barres de quelques pas), surveillance périodique des grappes par CND, remplacement systématique des grappes sur un critère de rebut; ii) l'étude de solutions "matériau », par traitement de surface ou revêtement, pour empêcher l'usure ; iii) le lancement de nombreuses actions pour comprendre, modéliser, prédire, éviter... [5], [6].

Concernant iii), les difficultés ont été nettement sous-estimées. Une $1^{\text {ère }}$ maquette hydraulique à échelle 1 ayant montré dès 88 que les crayons vibraient sous écoulements, la logique classique en vibration-usure du début des années 90 prévalut: a) identification des sources hydrauliques, b) quantification de ces excitations, c) calculs vibratoires non-linéaires (jeux) de la grappe soumise à ces excitations et déduction des puissances d'usure au droit des cartes:

$W_{R}=\frac{1}{T} \int f_{n} \cdot v_{t} \cdot d t, f_{n} \quad$ étant la force normale de contact, $\mathrm{v}_{\mathrm{t}}$

la vitesse tangentielle de glissement et $T$ le temps de scrutation, e) établissement en simulateur d'usure des coefficients d'usure $k, f$ ) estimation des volumes usés $V$ par la loi

\section{Détail du RIC.}

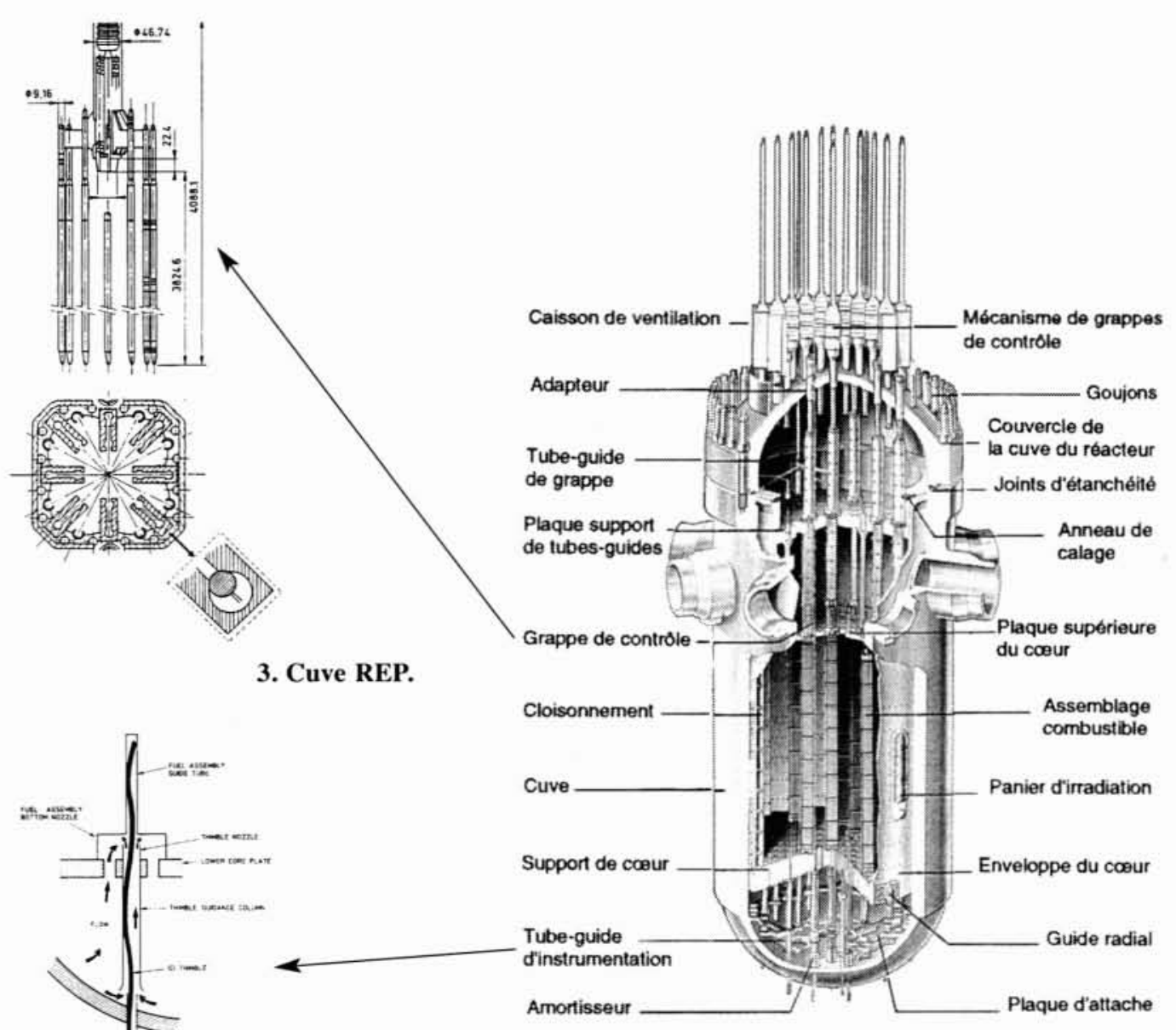


d'Archard: $\dot{V}=k \cdot W_{R}$. Cette démarche analytique, bien que remportant certains succès, ne permit pas d'expliquer de manière satisfaisante les usures trouvées en réacteur : répartition spatiale, discrimination suivant les crayons, cinétique d'usure, etc. De plus des expertises réalisées en 97 montraient que les faciès d'usure des crayons en réacteur différaient notablement de ceux trouvés en simulateur, ce qui signifiait que les vrais mécanismes d'usure n'étaient pas reproduits en machines de laboratoire. A décharge de ce constat, on notera que ce problème est formidablement complexe par la géométrie des grappes et leurs points de contact erratiques avec les systèmes de guidage, la nature des écoulements et des mécanismes d'IFS, l'environnement physicochimique, et bien d'autres paramètres encore mal identifiés qui font que «certains réacteurs usent et d'autres pas " ! Signalons enfin que l'usure-sous-carte, même si elle n'est pas expliquée de manière satisfaisante, est résolue en introduisant depuis quelques années des grappes nitrurées (qui ne s'usent pas) en réacteur. La solution « matériau » a donc pour l'instant " gagné ».

\subsection{Le Réseau d'Instrumentation du Cour (RIC)}

Dans les cœurs REP, des détecteurs neutroniques sont insérés dans les assemblages combustible en se déplaçant dans des « doigts de gant ", longs tubes flexibles d'une quinzaine de mètres, de $7.5 \mathrm{~mm}$ de diamètre et $1 \mathrm{~mm}$ d'épaisseur, qui sortent de la cuve par le fond pour relier la salle d'instrumentation proche. Ces doigts de gant sont maintenus par un système de tubes-guides sur toute leur longueur (voir fig. 4). La fuite d'un doigt fut détectée à Paluel, tête de série des $1300 \mathrm{MWe}$, huit mois après son démarrage. Les inspections révélèrent aussitôt de nombreuses traces d'usure sur la plupart des doigts de gant en différents endroits (pénétration de fond de cuve, entrée combustible....). Ces usures furent attribuées à des phénomènes de vibrations sous écoulement, ce qui fut rapidement confirmé par maquette hydraulique. Une faible différence de pression aux extrémités de la colonne de guidage à l'intérieur de la cuve créait autour du doigt un écoulement axial confiné pouvant atteindre $10 \mathrm{~m} / \mathrm{s}$, configuration propice à des phénomènes aigus d'IFS [7]. Une zone particulièrement suspectée était la buse en sortie de colonne, sous le pied d'assemblage combustible. Les études menées [8] confirmèrent des résultats antérieurs propres aux AGR (Advanced Gas Reactor), à savoir l'extrême sensibilité, en annulaire confiné, de l'angle du diffuseur de sortie sur les vibrations induites. Dans le cas présent, une buse présentant un (demi-) angle de $15^{\circ}$ générait des vibrations importantes, alors qu'une buse carrée (angle nul) les réduisait significativement, et ceci d'autant plus qu'elle était pourvue de fentes radiales qui "cassaient " les recirculations. L'analyse de ces phénomènes était néanmoins grandement compliquée par les conditions d'appui du doigt de gant à l'intérieur de la colonne de guidage, qui variaient au cas par cas vu la souplesse du doigt.

Contrairement au cas des grappes, le remède ne fut pas d'ordre «matériau», mais géométrique. En augmentant le diamètre des doigts de gant, en prolongeant les « bonnes buses " d'un chemisage à l'intérieur des colonnes de guidage, on réduisit le jeu annulaire, ce qui, par l'accroissement des pertes de charge, réduisit les vitesses axiales et les vibrations induites (sans compter l'influence des points de contact). Le retour d'expérience confirma le bien-fondé de cette approche (non évidente, des jeux réduits pouvant accentuer les phénomènes d'IFS et l'usure).

\section{VII —CALCULS ACCIDENTELS D'IFS}

La doctrine de la Sûreté nucléaire conduit à étudier toutes sortes de situations accidentelles, hautement improbables, dont on veut néanmoins analyser les conséquences dans le but de vérifier qu'elles n'induisent pas de situation encore plus critique. Citons ainsi, pour les RNR, l'Accident de Confinement $(\mathrm{ADC})$ dans la cuve suite à une réaction entre le sodium et le combustible provoquée (par ex.) par la fissuration d'une gaine, ou la Réaction Sodium-Eau (RSE) dans un générateur de vapeur secondaire suite à la rupture d'un tube GV ; pour les REP, l'Accident par Perte de Refroidissement Primaire (APRP) correspondant à la rupture guillotine quasi-instantanée d'une tuyauterie primaire (à 155 bar), ou l'explosion-vapeur suite à l'écoulement de corium (cœur fondu) dans l'eau restant en fond de cuve, en cas d'accident grave. Tous ces accidents, dont la liste n'est pas exhaustive, sont extrêmement violents et de courte durée dans le sens où ils engendrent les dommages mécaniques maxima dans les quelques dizaines, voire centaines, de millisecondes après leur initiation.

Leur modélisation requiert généralement un code de calcul de dynamique rapide, tel PLEXUS développé au CEA/Saclay [9]. PLEXUS traite, directement et complètement, des fluides et des structures, et donc de I'IFS. L'initiation de l'accident est modélisée par une injection localisée d'énergie (explosion-vapeur) ou par l'expansion hors équilibre d'une bulle de gaz (ADC, RSE) ou par la rupture d'un état d'équilibre de fonctionnement (APRP). L'IFS y est résolue par des méthodes avancées, comme l'ALE (Arbitrary Lagrangian-Eulerian) qui permet, par exemple, au maillage fluide de "suivre " la déformée de la structure. Résoudre directement le problème couplé fluide-structure permet d'établir des solutions de référence. Ces situations accidentelles sont en effet souvent modélisées en deux temps : i) calcul fluide de l'accident en considérant les parois raides, ii) calcul des structures (souples) en injectant aux parois les efforts dus au fluide déterminés précédemment. Les nonlinéarités du problème rendent cette approche de sous-structuration inexacte et souvent très pénalisante. Cette « marge " dans le calcul n'est pas toujours souhaitée, ou tout du moins doit être clairement identifiée, vu la situation hautement hypothétique analysée. Insistons enfin sur la finalité de tels calculs qui servent à démontrer que "les structures tiennent " : un APRP ne doit pas engendrer un autre APRP, l'enveloppe de cuve doit rester intègre en cas d'ADC,... La partie stratégique dans I'IFS reste donc la structure et les dommages mécaniques qu'elle subit, ce qui explique que l'IFS est parfois traitée « vu de la structure ».

\section{VIII — PROSPECTIVE 2000-2010}

Faire de la prospective est un exercice toujours délicat et risqué. On peut néanmoins le tenter à la lumière des expériences passées. Mieux appréhender les problématiques liées à l'IFS dans leur globalité sera sans doute un enjeu majeur de la prochaine décennie. Ainsi les spécialistes d'IFS devront, plus qu'aujourd'hui, mettre leur contribution en perspective, et non se cantonner dans leur domaine de prédilection souvent réducteur. La finalité de l'IFS dans les réacteurs nucléaires réside dans les dommages mécaniques qui en découlent. Or ces dommages peuvent apparaitre plus couplés qu'on ne le pensait à d'autres domaines (physico-chimie, matériau, etc.), comme l'illustre l'usure des grappes. Etre spécialiste en IFS tout en ayant une vue généraliste (matériel, fonctionnement, matériaux, etc.), et savoir travailler en synergie pour être plus efficace dans sa partie, voilà bien un défi des années futures, mais qui n'est pas propre à l'IFS.

Concernant les configurations connues d'IFS, comme les faisceaux de tubes (ex. des GV) ou les écoulements axiaux confinés (ex. du RIC), il s'agit dans le futur de comprendre en profondeur les mécanismes physiques d'interaction, ce dont on est loin aujourd'hui. Cette recherche amont, en rup- 
ture d'une certaine pratique actuelle, s'appuiera en partie sur les progrès des simulations numériques, sans toutefois surévaluer leur apport. Mais savoir calculer finement l'écoulement, stationnaire ou instationnaire, autour d'obstacles sera toujours « un plus » pour comprendre. Cette compréhension fine de l'IFS doit se retrouver intégrée dans des règles de construction, simples comme toute règle - du style carte de Connors - mais aux marges clairement démontrées et identifiées. Notons également qu'une attention particulière devra être portée au diphasique, si courant dans I'industrie, mais d'une difficulté d'ordre supérieur par rapport au monophasique.

Le CEA, par ses missions, notamment celle de fournir à la Puissance Publique les éléments pertinents de l'option nucléaire quand se posera le problème du renouvellement du parc vers 2010, est, et sera amené à étudier toute sorte de réacteur (HTR, Hybride, Rapide,....). Il s'agira devant ces dessins nouveaux et en l'absence de tout retour d'expérience, d'anticiper les problèmes d'IFS, et de « prévenir plutôt que de guérir » (voir l'ex. de Superphénix). Pour cela il faudra disposer, non seulement d'ingénieurs compétents en IFS, mais aussi de logiciels "boîte à outils", comme aujourd'hui CASTEM 2000 développé au CEA/Saclay, afin de pouvoir développer rapidement les applications d'IFS dont on n'a pas encore idée aujourd'hui. D'une manière générale, l'IFS devra profiter de toutes les avancées en analyse des structures et des fluides, du traitement des problèmes couplés aux aspects probabilistes, sans oublier les progrès en algorithmique. Le recours à des maquettes sera encore indispensable, mais sans doute plus limité et bénéficiant, là aussi, de tous les progrès en expérimental (mesures, plan d'expérience, fluide de substitution, etc.).

Enfin, et ce n'est pas le moins important, le Nucléaire tend à se normaliser par rapport aux autres industries en devant démontrer et accroître sa compétitivité. Dans ce climat de " moins d'argent », les échanges avec le Non-Nucléaire, déjà commencés, se poursuivront et se renforceront. Travailler en meilleure synergie avec tous les acteurs en IFS entre naturel- lement dans ce cadre, auquel ce colloque contribue significativement.

\section{RÉFÉRENCES}

[1] AxISA F.: " Flow-Induced Vibration of Nuclear System Components », in Technology for the 90's, ASME, 93.

[2] Arta S., Gibert R.J. : «Fluidelastic instability in a flexible weir : a theoritical model. ASME, PVP vol.104, 1986.

[3] Granger S., De Langre E. : " Vibrations sous écoulements et instabilités dynamiques des faisceaux de tubes des échangeurs de chaleur ". Revue Française de Mécanique, (1) : 38-47, 1995.

[4] Gibert R.J.: « Vibrations des structures. Interactions avec les fluides. Sources d'excitation aléatoires ». Paris. Editions Eyrolles, 1988.

[5] Borsot L., Canteneur Ch., Barbe G. : « Experimental and computational investigation into vibration wear of control rods in french PWRs ». $4^{\text {th }}$ International Symposium on FSI, AE, FIV \& Noise (ASME) ; Nov. 1997; Dallas, Texas.

[6] ZBinden M., LiNA A. : «L'usure dans les réacteurs à eau pressurisée : le cas des grappes de commande ». Epure n ${ }^{\circ} 63$, Juillet 1999.

[7] De Langre E., Granger S. : “Couplage structure-écoulement axial confiné ». Revue Française de Mécanique, (1) : 49$55,1995$.

[8] Gorman D.J., Godon J.L., Planchard J. : « Analytical and experimental study of the vibratory response of a flexible tube subjected to external annular flow part away along its length ». Int.Conf. on FIV (BHRA), May 1987, England.

[9] Lepareux M., Bung H.: «PLEXUS, a general computer code for fast dynamic analysis ». SMIRT 8 (1985). 